\title{
Modelling the Volatility of Central Bank of Kenya Currency Exchange Rates
}

\author{
Oganga Caneble*, Anthony Wanjoya, Anthony Ngunyi \\ Department of Statistics and Actuarial Sciences, Jomo Kenyatta University of Agriculture and Technology, Nairobi, Kenya \\ Email address: \\ canebleoganga@gmail.com (O. Caneble), awanjoya@gmail.com (A. Wanjoya), anthonyngunyi@gmail.com (A. Ngunyi) \\ ${ }^{*}$ Corresponding author
}

\section{To cite this article:}

Oganga Caneble, Anthony Wanjoya, Anthony Ngunyi. Modelling the Volatility of Central Bank of Kenya Currency Exchange Rates. International Journal on Data Science and Technology. Vol. 7, No. 3, 2021, pp. 62-68. doi: 10.11648/j.ijdst.20210703.13

Received: August 15, 2021; Accepted: August 25, 2021; Published: September 4, 2021

\begin{abstract}
In emerging countries, such as Kenya, the foreign exchange market is an important aspect in the economic development of a country. The currency exchange rate market, like the rest of the world's financial markets, has been marked by considerable instabilities over the last decade. The objective of this paper is to model the volatility of the KSH/USD exchange rate prices using and calculate the VaR using the GARCH-EVT model. In particular, this article uses the two-stage GARCH-EVT approach to estimate the value at risk of the Kenyan Shilling against the US dollar., particularly the one-day ahead Value-at-Risk forecast in risk control. The conditional and unconditional coverage test are used to back test the model. We compare the performance of the GARCH-EVT with the daily log returns of key currency in addition to modelling the value at risk in the Kenyan Foreign Exchange market (US dollar) foreign currencies from the period November 2004 - June 2021 for trading days with the exception of holidays and weekends. The mean equation that was best fitting for the data was ARMA $(4,2)$. The optimal GARCH model for the returns of the KSH/USD exchange rate is the GARCH $(1,3)$ with student-t innovations. The results of the backtesting show that GARCH-EVT can be utilized to estimate and forecast VaR at both 5\% and $1 \%$ level of significance.
\end{abstract}

Keywords: Value-at-Risk, Extreme Value Theory, GARCH and Backtesting

\section{Introduction}

Modelling volatility has been an immense field of research in observational fund with applications extending from resource evaluating, portfolio assignment, subsidiary estimating, all through to hazard the executives. Volatility alludes to the extent of changes in the arrival of a benefit. That volatility is the unconditional variance of returns provided by an asset. Variance or standard deviation is used as a measure of risk in risk management hence volatility can be termed as the amount of uncertainty or risk on the return of a given security. Higher volatility demonstrates that arrival on a security is spread over an enormous interim while lower unpredictability suggests return shifts over a little range or there are no evident extraordinary variances. The reason for volatility in any money related market is simply the exchanging. Financial time series data are built on the premise of volatility, that is, volatility defines financial time series data.
The modelling and forecasting of exchange rates and their volatility has important implications for many issues in economics and financial market. Volatility needs monitoring and presents a major risk to investors and policy makers. Many models have been applied in modelling the volatility of exchange rates in various countries and has been found that different models fit different data.

How to estimate and quantify market risk, particularly in financial markets, has become a key issue in recent years. Because of its simplicity among the risk metrics available, Value-at-Risk (VaR) has been widely employed to quantify hazards. VaR as a way to measure risk of a financial asset was proposed and applied by Jorion P since the challenge of quantifying risk existed [1]. Value-at-Risk is defined as the worst loss that can happen with a given confidence level over a given time [2].

The analytical and historical approaches to estimating VaR are the two main approaches. In the former, the returns are assumed to have a known distribution. The distribution of the 
returns is modelled in part due to serial autocorrelation and heterogeneity of the returns. The historical approach avoids making assumptions about distribution and instead focuses on the empirical distribution of prior data. The key disadvantage of this strategy is the selection of an adequate window size and the excessive reliance on previous data.

To estimate VaR across different locations and countries, a variety of models have been presented. Daily asset returns are supposed to follow a normal distribution in much of the literature, however they are leptokurtic and skewed that can cause the Value-at-Risk to be exaggerated or underestimated. The GARCH (Generalized Autoregressive Conditional Heteroscedasticity) and conditional Extreme Value Theory models have lately gained popularity. In light of this, the study investigated the features of the Kenyan Foreign Exchange Market, using a variety of models.

The main purpose of this study is to better understand the major strengths and limits of GARCH model and Extreme Value Theory in estimating the value at risk. The Kenyan foreign exchange market is of relevance to us for this project. The US Dollar was utilized as the currency. The Kenya Shilling has declined against the US Dollar in recent years, particularly during the fiscal year 2015/2016, owing to tighter global financial market conditions and China's recession. Backtesting tests are used to assess how well the model fared in calculating the Value-at-Risk.

The remainder of the paper is divided into the following sections: Part 2 delves into previous research, while Part 3 delves into the GARCH family of models and Extreme Value Theory, as well as the basic calculations for estimating the Value-at-Risk using the Extreme Value Theory model. Part 4 discusses the exchange rate that was employed in this project. The general results are presented in Part 5. Backtesting is covered in Part 6. Lastly, part 7 gives the conclusion and areas to be considered for further studies.

\section{Literature Review}

The GARCH model is the most widely used parametric approach for modelling time-varying volatility in various distributions. GARCH models are based on different distributions, such as the normal, student-t, and skewed student-t distributions. The GARCH with normal distribution, on the other hand, has been strongly chastised for underestimating risk. Because of this GARCH-normal shortcoming, the GARCH that incorporates student $t$ innovations was proposed to address the aspect of heavy tails in financial data.

The heteroscedasticity in outside trade for US, UK, Euro and Japanese Yen information has been modelled using GARCH models [3]. Month to month midpoints for the different money trade rates were gathered for the period from January 2001 to December 2010, an aggregate of 120 perceptions for every outside cash. The period was picked in view of the two significant achievements that the nation experienced i.e., Political decision period followed by the post-political decision viciousness in 2007/2008. Their significant point is to consider how these occasions influenced the presentation of the afore mentioned currencies.

For contrast, the EGARCH model has been used to model USDKES, EURKES, and GBPKES exchange rate volatility under the assumptions of both normal and student- $t$ distributions [4]. He noted that the student-t EGARCH is preferable to the normal distribution due to evidence of the financial time series' heavy tailed character. According to the authors, the normal GARCH model could neither explain the entire fat tail nature of the data nor could it explain the asymmetric responses. He then goes ahead to describe the EGARCH model as well as to give a specification of the two error distributions i.e., normal and student-t.

The student-t and GED distribution to model the innovations of the Naira-USD exchange rate [5]. Some of the shortcomings they saw are that the authors considered monthly data in their investigations and based on this; the series' characteristics were not well captured. Also, the studies only considered one or two exchange rates out of many. Other than that, the studies assumed a normal distribution and did not look at different distributional forms. Lastly, they felt that due to the volatility and asymmetry in exchange rate series, daily data should have been applied to examine these properties.

GARCH model has been utilized in the estimation of unpredictability in the Kenyan outside trade showcase data for the period of 1993-2006 [6]. In their analysis they found that exchange rates are Leptokurtic and slightly positively skewed. The estimated models fitted the data well, confirming that GARCH $(1,1)$ is adequate in describing volatility in many financial time series as claimed by [7].

The Chinese Yuan (CNY) Currency risk was forecasted using the extreme value theory by [8]. The authors sought a perfect method to measure the tail risk in the CNY foreign exchange rate market. Various studies have estimated the value at risk in Rwanda Exchange Rate [9]. The extreme volatility in the daily exchange rates of Kenya Shillings against the US dollar has been modelled by [10]. The authors applied a peak over threshold and determined that despite episodes of extreme volatility; long-term stability was maintained over the period 1999 to 2013 .

\section{Methodology}

\subsection{Volatility}

The dispersion of all likely outcomes of an uncertain variable is referred to as volatility. Typically, we are concerned in the distribution of asset returns in Financial Markets. Mathematically, volatility is defined as:

$$
\sigma=\sqrt{\frac{1}{T-1} \sum_{t=1}^{T}\left(r_{t}-\mu\right)}
$$

Where $\sigma$ is the standard deviation, $r_{t}$ is the return on day $\mathrm{t}$ and $\mu$ is the average return over the return T-day period. 


\subsection{Volatility Models}

Volatility models captures the time varying conditional variance in financial time series. The $\log$ returns decomposition is defined by:

$$
r_{t}=\mu_{t}+y_{t}, y_{t}=\sigma_{t} e_{t}
$$

Where $\mu_{t}$ is the mean component and $y_{t}$ is the conditional volatility. $\sigma_{t}$ is the shock of an asset at time t.

\subsubsection{Mean Component}

To model the mean component of a financial time series using the Autoregressive Moving Average process. The ARMA model is a linear combination of the Autoregressive model and the Moving Average model. The ARMA model of order $(p, q)$ is given by:

$$
X_{t}=\mu+\sum_{i=1}^{p} \theta_{i}\left(y_{t}-\mu\right)+\sum_{j=1}^{q} \theta_{j}\left(e_{t-j}\right)+e_{t}
$$

Where $y_{t}-\mu$ is the lagged returns at time t. $X_{t}$ is the exchange rate return at time $\mathrm{t}, \mu$ is the mean, $\theta_{i}$ and $\theta_{j}$ are weights and $e_{t}$ is the error at time t, $e_{t-j}$ is the past error terms.

\subsubsection{Standard GARCH Model}

Because linear models fail to explain a number of fundamental properties found in many financial data sets, such as leptokurtic, volatility clustering, long memory, and leverage effects, researchers have devised models to handle financial time series data. The GARCH model that was proposed by [7] is described in this section.

The general form of the GARCH (p, q) model is given by:

$$
r_{t}=\mu+a_{t}, a_{t}=\sigma_{t} \varepsilon_{t}
$$

where $\delta_{t}$ is given by

$$
\sigma_{t}^{2}=\alpha_{0}+\sum_{i=1}^{p} \alpha_{i} \alpha_{t-i}^{2}+\sum_{i=1}^{q} \beta_{i} \delta_{t-j}^{2}
$$

Where $r_{t}$ denotes the log returns, $\mu$ are the mean, $a_{t}$ are the innovations from the mean equation. To guarantee stationarity of the series, $\alpha_{1}+\beta_{1}<1$.

\subsection{Conditional Distributions}

The prevalence of fat-tails in financial data necessitates the employment of alternative distributions such as the student $t$ distribution and skewed student t-distribution since returns are not normally distributed. In this paper, the normal distribution, student $t$-distribution were used in the GARCH models and based on the model with smallest AIC value, the selection was made for the optimal model.

\subsubsection{Student-t Distribution}

The student $\mathrm{t}$ distribution whose form is given in the equation was introduced by [7]:

$$
f\left(z_{t}, t\right)=\frac{\Gamma\left(\frac{v+1}{2}\right)}{\Gamma\left(\frac{v}{2}\right) \sqrt{\pi(v-2)}}\left(1+\frac{z_{t}^{2}}{v-2}\right)^{-\frac{v+1}{2}}
$$

Where $\Gamma($.$) is the gamma function.$

\subsubsection{Normal Distribution}

The normal distribution according to [11] is denoted by:

$$
f\left(z_{t}\right)=\frac{1}{\sqrt{2} \pi} e^{\frac{z_{t}^{2}}{2}}
$$

\subsection{Extreme Value Theory}

Extreme value theory, unlike the GARCH model, only considers the tail of the distribution. In general, two ways to modelling extremes have been presented. The block maxima method developed by [12] is one of them. The largest observation in the blocks is assumed to be an extreme value in this procedure. The peak over threshold approach is the second method, in which an extreme value is an observation above a certain threshold.

Extreme events are defined as observations which surpass a specific threshold $u$ in the peak over threshold approach. Given a random variable $Z$, the excess distribution function above a certain threshold $u$ is expressed as

$$
F_{u}(x)=P[Z-u \leq x \mid Z>u]
$$

Where $\mathrm{x}$ is the excess of $\mathrm{Z}$ over threshold $\mathrm{u}$. If $Z-u \geq 0$, the excess function can be written as:

$$
F_{u}(x)=\frac{F(x+u)-F(u)}{1-F(u)}
$$

Let $\mathrm{F}$ be an underlying distribution that describe an entire time series $0 \leq x<Z_{F}-u$ Where $Z_{F}-u$ is the right end point of $F$. The interest is to estimate the defined by $F_{u}$. [13] and [14] proved that the excess distribution function $F_{u}(x)$ is well approximated by the Generalized Pareto Distribution for a large class of underlying distribution functions.

$$
F_{u}(x) \approx G_{\xi, \beta}(x) ; u \rightarrow \infty
$$

Where

$$
G_{\xi, \beta}(x)=\left\{\begin{array}{r}
1-\left(1+\frac{\xi \beta}{x}\right)^{-\frac{1}{\xi}}, \xi \neq 0 \\
1-e^{\frac{-x}{\beta}}, \xi=0
\end{array}\right.
$$

For $x \in\left[0, x_{F}-u\right]$ if $\xi \geq 0$ and $y \in\left[0, \frac{-\beta}{\xi}\right]$ if $\xi<0$.

The choice of a threshold in the peak over threshold approach is critical. The graphical representation, which uses the mean excess graph, is one approach of selecting a threshold. In this study, the mean excess function is used, and the threshold, $u$, is set based on the observed mean excess being almost linear. 


\subsection{Value-at-Risk Estimation under EVT}

In this section, in order to estimate the Value-at-Risk the study follows the procedure described in [15]. A summary of the two steps that were implemented in this paper are described as:

i. The residuals from the estimated GARCH model (GARCH $(1,3)$ in this case) are extracted.

ii. The residuals are used in fitting the EVT model and the estimates of the Value-at-Risk derived.

After selecting the threshold one can use the GPD in order to model the extracted residuals and use the following description to calculate the VaR estimates:

$$
\operatorname{VaR}_{q}=u+\frac{\beta}{\xi}\left[\left(\frac{n}{N_{u}}(1-q)^{-\xi}\right)-1\right]
$$

Where $\mathrm{n}$ is the sample size and $N_{u}$ is the observations above the threshold.

\section{Data Description}

The analysis has been done using average monthly exchange rates for the following currency pairs: Kenya Shillings against US Dollars. The choice of these currency was based on its relative proportions, in the Bank's foreign exchange investment portfolio and based also on their currency composition of the Kenyan imports. The daily exchange rate of Kenya shilling against US Dollar for the period of 2nd November 2004 to 30th June 2021 was used. The data was obtained from Central Bank of Kenya website. The total observations are 4195 daily returns for the USD/KES exchange currency excluding the holidays and weekends

In order to transform the currency exchange rates into log returns the following formula was used.

$$
R_{t}=\log \left(\frac{P_{t}}{P_{t-1}}\right)
$$

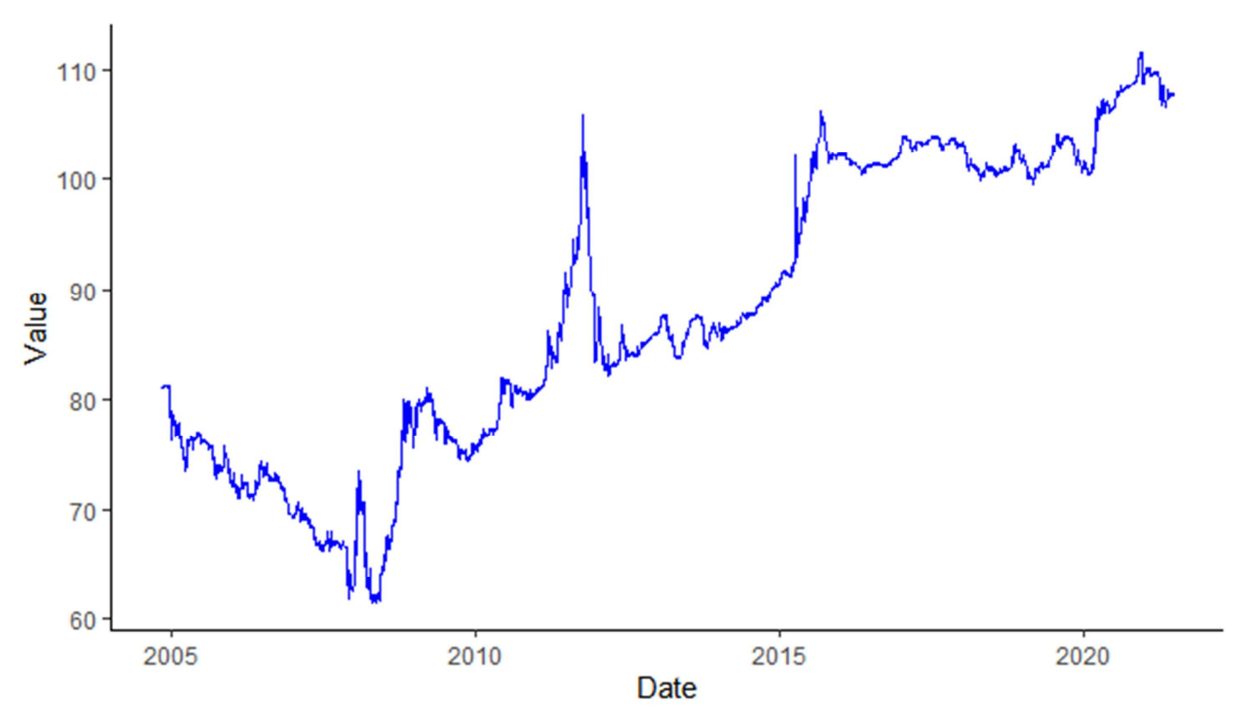

Figure 1. Plot for KSH/USD daily exchange rate prices.

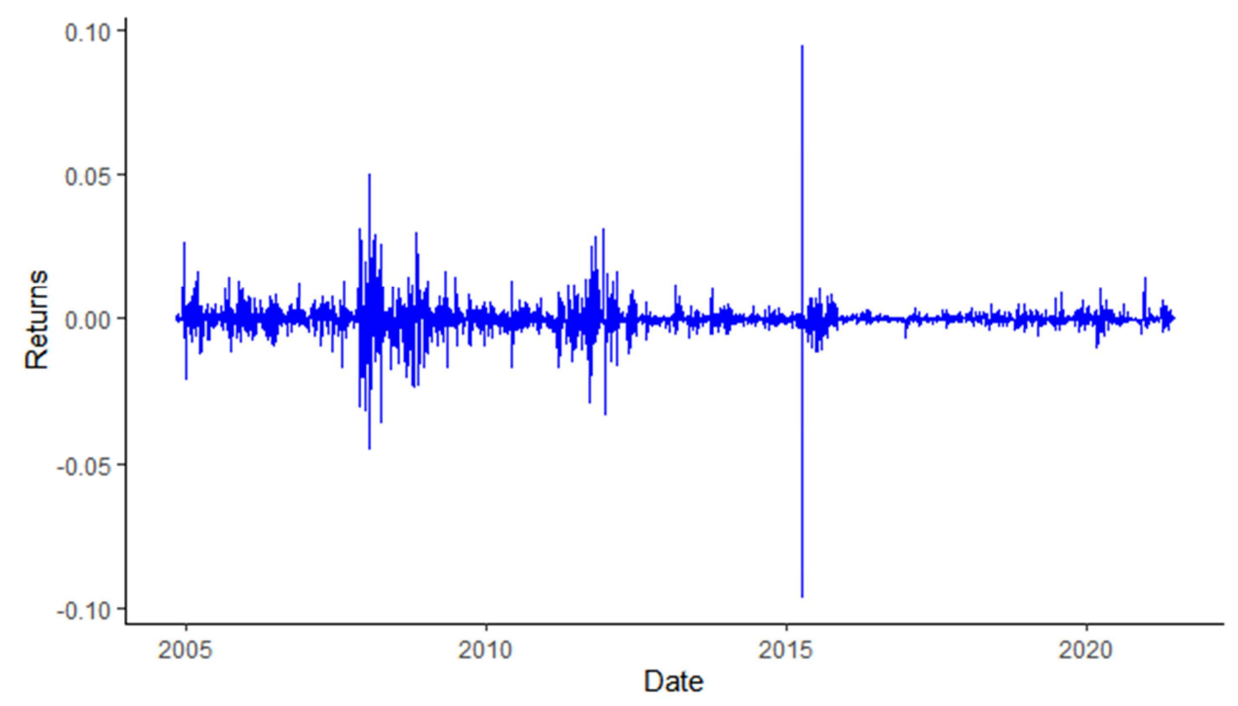

Figure 2. Plot for daily returns for USD/ KSH. 


\section{Result}

The raw prices for the Kenya Shillings versus US Dollar are displayed in Figure 1. It can be observed that between the years 2005 and 2007 there was a decline in the exchange rate prices. In 2008, a sharp decline was observed which is attributed to the occurrence of post-election violence that befall the country. From 2016 to the year 2017 only a few weeks before the general elections there was great stability in the prices. However, after the elections in 2017 the prices declined due to unfavourable political climate. As observed in Figure 2, the daily log returns indicate a period of high volatility and as indicated there is volatility clustering which corroborates further the high volatility of developing countries financial market.

Table 1. Summary Statistics for Exchange Rate Returns.

\begin{tabular}{ll}
\hline Statistic & US Dollar \\
\hline Minimum & -0.095776 \\
Mean & -0.000192 \\
Maximum & 0.094458 \\
Variance & 0.000021 \\
Skewness & -0.037304 \\
Kurtosis & 105.803208 \\
Jarque-Bera & 1958127.9729 \\
JB p-value & $2.22 * 10^{-16}$ \\
\hline
\end{tabular}

Table 1 shows summary statistics of the daily returns of exchange rates. The negative loss implies that there is an upward movement of foreign exchange. The exchange rate for US Dollar has a more positive shock. The high value of kurtosis indicates that the attribute of financial data of having heavy tails. As the test for normality the $p$ values was small hence further giving weight to the non-normality of returns.

The Augmented Dicky fuller test was used to check for stationarity of the rate returns. The $\mathrm{p}$ values were found to be 0.01 so the null hypothesis of non-stationarity was rejected and concluded that the returns were stationary.

Table 2. Criteria for ARMA (p, q) order selection.

\begin{tabular}{lll}
\hline Model & AIC & Log Likelihood \\
\hline ARMA $(0,0)$ & -33285.16 & 16644.58 \\
ARMA $(0,1)$ & -33315.49 & 16660.75 \\
ARMA $(1,0)$ & -33310.42 & 16658.21 \\
ARMA $(1,1)$ & -33323.11 & 16665.56 \\
ARMA $(1,2)$ & -33332.95 & 16671.47 \\
ARMA $(2,1)$ & -33336.05 & 16673.03 \\
ARMA $(2,0)$ & -33337.97 & 16672.99 \\
ARMA $(0,2)$ & -33333.4 & 16670.7 \\
ARMA $(2,2)$ & -333345.8 & 16678.9 \\
ARMA $(2,3)$ & -33346.35 & 16680.17 \\
ARMA $(2,4)$ & -33351.95 & 16683.98 \\
ARMA $(3,0)$ & -33336.14 & 16673.07 \\
ARMA $(3,2)$ & -33356.97 & 16685.48 \\
ARMA $(3,3)$ & -33343.38 & 16679.69 \\
ARMA $(3,4)$ & -33360.32 & 16689.16 \\
ARMA $(4,0)$ & -33343.41 & 16677.71 \\
ARMA $(4,1)$ & -33357.65 & 16685.82 \\
ARMA $(4,2)$ & -33362.36 & 16689.18 \\
ARMA $(4,3)$ & -33360.34 & 16689.17 \\
ARMA (4,4) & -33358.21 & 16689.1 \\
\hline
\end{tabular}

Table 2 shows the log returns' optimal mean equation. The distribution with the lowest AIC score was chosen as the optimal mean equation. The ARMA $(4,2)$ model with student $t$ distribution is the best ARMA model for the USD/KSHS.

The Box-Ljung test was used to check for model adequacy of the model. The null hypothesis is that the model was adequate versus the alternative that the model is not adequate. The results are presented in Table 3. As indicated the $p$ value is bigger than 0.05 the level of significance, hence the model is adequate.

Table 3. Ljung-Box test for Model adequacy.

\begin{tabular}{lll}
\hline Chi-square & df & pvalue \\
\hline 0.0000379 & 1 & 0.9951 \\
\hline
\end{tabular}

The Box-Ljung test was used to check for ARCH effects. The null hypothesis of the Box-Ljung test is that there are no $\mathrm{ARCH}$ effects and the alternative hypothesis is that there are ARCH effects. Using the optimal ARMA model and the squared standardized residuals the results are presented in Table 4. As indicated the $\mathrm{p}$ value was smaller than 0.05 hence, we conclude there are ARCH effects thus we model using GARCH models.

Table 4. Ljung-Box test for Model adequacy.

\begin{tabular}{lll}
\hline Chi-square & df & pvalue \\
\hline 10.528 & 1 & 0.001176 \\
\hline
\end{tabular}

The ACF and the PACF plots for the squared residuals are presented in Figure 3. As indicated there is presence of serial autocorrelation.
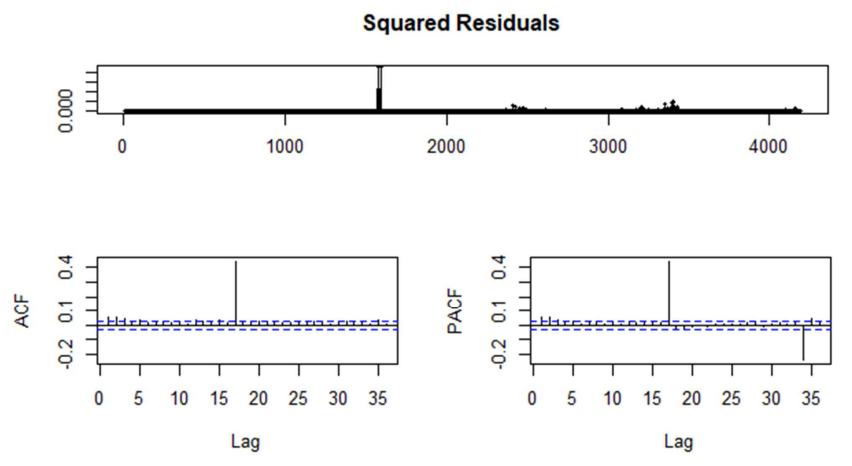

Figure 3. ACF and PACF of squared residuals.

GARCH estimation was performed and the optimal model combination was the ARMA $(4,2)-\operatorname{GARCH}(1,3)$ as indicated in Table 5. The selection was based on the model with the smallest values of Akaike Information Criterion.

Table 5. Criteria for ARMA $(p, q)-G A R C H(p, q)$ order selection.

\begin{tabular}{lll}
\hline Model & AIC & Log Likelihood \\
\hline ARMA (4,2)-GARCH $(1,1)$ & -8.642489 & 18131.3 \\
ARMA (4,2)-GARCH $(1,2)$ & -8.642004 & 18131.28 \\
ARMA (4,2)-GARCH $(2,1)$ & -8.890673 & 18652.7 \\
ARMA $(4,2)-G A R C H(2,2)$ & -8.701029 & 18256.06 \\
ARMA $(4,2)-G A R C H(1,3)$ & -8.928086 & 18732.2 \\
\hline
\end{tabular}


Table 6. Criteria for ARMA $(4,2)-G A R C H(1,3)$ model with different conditional distributions.

\begin{tabular}{llll}
\hline & Normal & Skewed Student-t & Student-t \\
\hline AIC & -8.928086 & -9.611322 & -9.61162 \\
Log Likelihood & 18732.2 & 20166.94 & 20169.57 \\
\hline
\end{tabular}

To check the best error distribution the study fitted data to the optimal ARMA-GARCH model. The model with student$\mathrm{t}$ distribution was the best fitting model.

The parameter estimates of the fitted model was obtained and the results presented in Table 7. The shape parameter is significant and it demonstrates the heavy tail of the distribution which characterizes financial data.

Table 7. Summary for parameter estimates.

\begin{tabular}{lll}
\hline Parameter & Estimate & p-value \\
\hline$\mu$ & 0.00005678 & 0.0000285 \\
$\operatorname{ar} 1$ & 0.1864 & $<2 * 10^{-16}$ \\
\hline
\end{tabular}
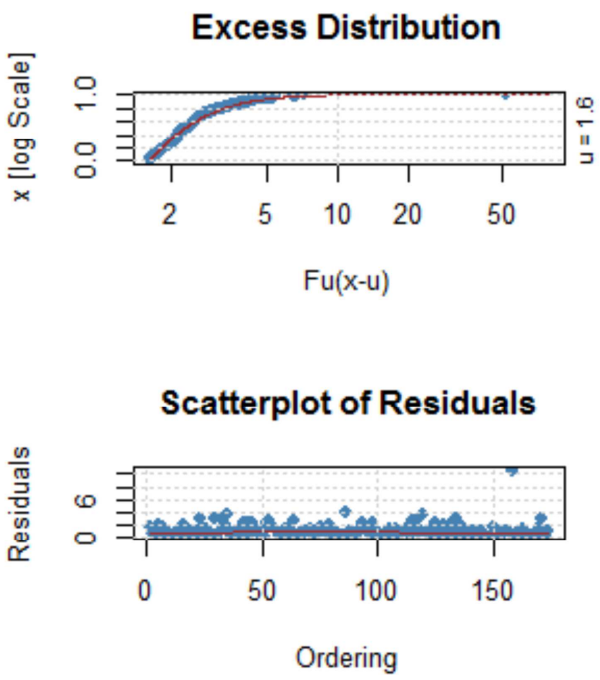

\begin{tabular}{lll}
\hline Parameter & Estimate & p-value \\
\hline ar2 & -0.02469 & 0.086986 \\
ar3 & -0.09697 & 0.452226 \\
ar4 & -0.1491 & 0.24549 \\
$\Pi$ & 0.0000000126 & 0.000383 \\
$\beta_{1}$ & 1 & $3.05 \mathrm{e}-08$ \\
$\beta_{2}$ & 0.4629 & $8.42 \mathrm{e}-08$ \\
$\beta_{3}$ & 0.00000001 & 1 \\
$\beta_{4}$ & 0.1757 & 0.005581 \\
Shape & 2.375 & $<2 * 10^{-16}$ \\
\hline
\end{tabular}

Extreme Value Theory

To investigate if the excess distribution, follows a generalized Pareto distribution, Figure 4 illustrates the empirical excess distribution as well as the residuals QQ-Plot. The plot show that the empirical excess distribution follows GPD throughout the exchange rates, meaning that the exceedances may be represented by GPD.
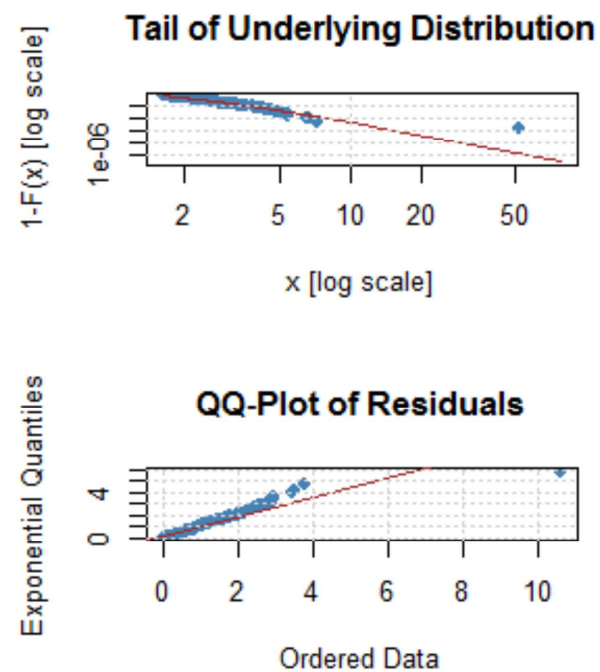

Figure 4. Diagnostic Check for USD/KSHS Exchange Rate.

Table 8 shows the predicted parameter for US Dollar against the Kenya Shillings, as well as the related thresholds. The $\xi$ denotes the estimated parameters that determine the distribution's type. Because it is positive, this indicates that the selected currency's distribution corresponds to the Fréchet distribution's maximal domain of attraction, which is heavytailed, [14].

Table 8. Model Parameters for GPD

\begin{tabular}{llll}
\hline Threshold $(\boldsymbol{\tau})$ & $\boldsymbol{\xi}$ & $\boldsymbol{\beta}$ & No. of Exceedances \\
\hline 0.02 & 0.0069144 & 0.41833 & 17 \\
\hline
\end{tabular}

\section{Backtesting of the VaR Models}

\subsection{VaR Forecasting}

The GARCH-EVT model in calculating the Value-at-Risk have been presented. The Value-at-Risk forecasts based on the model and the forecasting results are presented in this part. The unconditional coverage test and conditional coverage test are presented in the next section.

\subsection{Unconditional and Conditional Coverage Test}

[16] proposed the unconditional coverage test, which is based on failure rates. The test determines whether the number of exceptions matches the level of confidence. To perform this test the required values are the number of observations (N), number of exceptions (y) and the confidence level (c). The main idea will be to find out whether the observed failure rate is significantly different from the failure rate suggested by the confidence level. The likelihood ratio statistic is given by:

$$
L R_{u c}=-2 \ln \left(\frac{(1-p)^{N-y} p^{y}}{\left[1-\frac{y}{N}\right]^{N-y}\left(\frac{y}{N}\right)^{y}}\right)
$$

The correct model is one that produces the appropriate number of violations. [17] created the conditional coverage test, which checks if the number of violations is the same as the expected one, and also the independence of failures over time. The likelihood ratio statistic is given by: 


$$
L R_{c c}=-2 \ln \left(\frac{(1-\pi)^{n_{00}+n_{01}} \pi^{n_{11}+n_{01}}}{\left(1-\pi_{0}\right)^{n_{00}} \pi_{0}^{n_{01}}\left(1-\pi_{1}\right)^{n_{10}} \pi_{1}^{n_{11}}}\right)
$$

Where $n_{i i}$ represents the number of observations with value I followed by $\mathrm{j}$ and $n_{i j}=\frac{n_{i j}}{\sum n_{i j}}$ is the corresponding probability.

\subsection{Backtesting Results}

A rolling window of 250 is used in order to facilitate the forecasting of one day ahead VaR with $1 \%$ and $5 \%$ level of significance. Therefore, the model is back tested at the $5 \%$ level of confidence. The results are presented in Table 9.

Table 9. Comparative results for forecasted VaR.

\begin{tabular}{lll}
\hline Model & & $\mathbf{1 \%}$ level of significance \\
\hline & Expected & 2.5 \\
& Observed & 7 \\
& Observed (\%) & 2.8 \\
GARCH-t & & $5 \%$ level of significance \\
& Expected & 12.5 \\
& Observed & 13 \\
& Observed (\%) & 5.2 \\
\hline
\end{tabular}

Table 10. Unconditional Coverage (UC) Test at various Confidence Level.

\begin{tabular}{lll}
\hline & $\mathbf{1 \%}$ & $\mathbf{5 \%}$ \\
\hline GARCH-t & 0.019 & 0.885 \\
\hline
\end{tabular}

Table 11 shows the results of the conditional coverage test. The null hypothesis is that the number of violations is correctly counted and that they are unrelated. The best model is the one that does not reject the null hypothesis. The results show that the model for the US Dollar is the GARCH model with student $t$ distribution.

Table 11. Conditional Coverage (CC) Test at 95\% Confidence Level.

\begin{tabular}{lll}
\hline & $\mathbf{1 \%}$ & $\mathbf{5 \%}$ \\
\hline GARCH-t & 0.025 & 0.918 \\
\hline
\end{tabular}

\section{Conclusion}

The goal of this article was to assess GARCH-EVT forecasts' Value-at-Risk estimates. The study's findings show that the GARCH-EVT with student $t$ distribution model can be utilized to estimate Value-at-Risk for the US Dollar exchange rate. The GARCH-EVT models are a good choice for forecasting VaR, according to the back testing results. For future works, an incorporation of a bigger rolling window can be used and number of currencies increased.

\section{References}

[1] P. Jorion, Value At Risk: The New Benchmark for managing Financial Risk, 2006.

[2] Jorion, Value at Risk The New Benchmark for Managing Financial Risk, McGraw-Hill, 2001.

[3] S. Omar, "GARCH modeling in monthly foreign exchange and share prices for specificcompanies in Kenya.," American Journal of Mathematics and Applications, vol. I, pp. 5-12, 2013.

[4] R. T. Kipkoech, "Modeling volatility under normal and student-t distributional assumptions (a case study of the Kenyan exchange rates)," American Journal of Applied Mathematics and Statistics, pp. 179-184, 2014.

[5] A. A. Adepoju, O. S. Yaya and O. O. Ojo, "Estimation of Garch models for Nigerian Exchange rates under nonGaussian innovations," 2013.

[6] I. Maana, P. N. Mwita and R. Odhiambo., "Modelling the volatility of exchange rates in the Kenyan market," African Journal of Business Management, 2010.

[7] Bollerslev, "Generalized Autoregressive Condtional Heteroscedasticity," Journal of Econometrics, 1986.

[8] Y. Lan, K. Chokethaworn and C. Chaiboonsri., "Forecasting Chinese Yuan currency risk with extreme value theory," 2014.

[9] J. de Dieu Ntawihebasenga, J. K. Mung'atu and P. N. Mwita, "Modeling the volatility of exchange rates in Rwandese markets.," American Journal of Theoretical and Applied Statistics, 2015.

[10] I. aana, A. Kamau and K. Kisinguh, "Modelling extreme volatility in the daily exchange rates of the Kenya shilling against the US dollar," Journal of Economics and International Finance, 2015.

[11] Engle, "Autoregressive Conditional Heteroscedasticty with estimates of variance of United Kingdom Inflation," Econometrica, pp. 987-1008, 1982.

[12] E. J. Gumbel, Statistics of Extremes, New York: Columbia University Press, 1958.

[13] A. A. Balkema and d. Haan, "Residual Life Time at Great Age," The Annals of Probability, vol. 2, no. 5, pp. 792-504, 1974.

[14] J. Picklands, "Statistical Inference Using Extreme Order Statistics," The Annals of Statistics, vol. 3, pp. 119-131, 1975.

[15] M. Alexander and Frey, "Estimation of Tail-Related Risk Measures for Heteroskedastic Financial Time Series: An Extreme Value Approach," Journal of Empirical Finance, vol. 7, pp. 271-300, 2000.

[16] P. Kupiec, "Techniques for Verifying the Accuracy of Risk Measurement Models," FEDS Paper, 1995.

[17] P. F. Christoffersen, "Evaluating Interval Forecasts," International Economic Review, vol. 39, pp. 841-862, 1998. 